Table. Platelet Aggregation Inhibition

\begin{tabular}{|c|c|c|c|c|c|c|}
\hline & Adenosine & Water Extract & Hexane Extract & Chloroform Extract & Ethanol Extract & Methanol Extract \\
\hline Inhibition (\%) & $68.6 \pm 4.3$ & $72.7 \pm 3.3$ & $40.1 \pm 5.6$ & $44.7 \pm 2.7$ & $43.5 \pm 6.6$ & $68.8 \pm 6.7$ \\
\hline
\end{tabular}

of $250 \mathrm{mg} / \mathrm{ml}$ showed a potent inhibition effect toward platelet aggregation. Compared with other extracts, water extract exhibited the strongest inhibition as well as adenosine $(50 \mathrm{uM} / \mathrm{ml})$ as a positive control.
In Addition to this, methanol extracts showed relatively similar inhibition pattern with adenosine. Finally, this initial finding has provided the evidence of Phyllantus niruri L. action in platelet functions.

\title{
PRODUCTION OF ANTI-MADECASSOSIDE POLYCLONAL ANTIBODY AND ITS APPLICATION FOR QUALITY CONTROL OF CENTELLA ASIATICA (L.) URBAN
}

\author{
(C) PutalunWaraporn ${ }^{1,2}$, Tassanawat Patcharin ${ }^{1,2}$, Yusakul Gorawit ${ }^{1,2}$, Sritularak \\ Boonchoo $^{3}$, Juengwatanatrakul Thaweesak ${ }^{4}$, Tanaka Hiroyuki ${ }^{5}$
}

\begin{abstract}
${ }^{1}$ Faculty of Pharmaceutical Sciences, Khon Kaen University, Khon Kaen, 40002, Thailand. ${ }^{2}$ Research Group for Pharmaceutical Activities of Natural Products using Pharmaceutical Biotechnology (PANPB), National Research UniversityKhon Kaen University, Khon Kaen 40002, Thailand. ${ }^{3}$ Faculty of Pharmaceutical Sciences, Chulalongkorn University, Bangkok 10330, Thailand. ${ }^{4}$ Faculty of Pharmaceutical Sciences, Ubon Ratchathani University, Thailand.

${ }^{5}$ Graduate School of Pharmaceutical Sciences, Kyushu University, Fukuoka 812-8582, Japan
\end{abstract}

Madecassoside is the major triterpenoid saponins derived from Centella asiatica (L.) Urban, a medicinal plant used in Ayurvedic medicine for centuries. Madecassoside possesses various biological activities, including antidepressant effect (1), burn wound healing and anti-inflammatory (2). In this study, we developed the immunoassay method for quantitative analysis of the major compounds in C. asiatica. A sensitive indirect competitive enzyme-linked immunosorbent assay (ELISA) was developed using polyclonal antibody against madecassoside. Madecassoside-bovine serum albumin conjugate was immunized to rabbits for producing polyclonal antibody. The results showed that the antibodies were specific for madecassoside and aisaticoside. The range of the assay extends from 0.39 to $50 \mu \mathrm{g} / \mathrm{ml}$. A good correlation between ELISA and HPLC methods was obtained when analysis of total triterpenoid saponins in the crude extracts of plant samples. In addition, the products containing $C$. asiatica in various preparations were determined triterpenoid saponins content by competitive ELISA. The developed ELISA method can be used for quality assessment of $C$. asiatica and their products. References: (1) Liu, M. R., Han, T., Chen, Y., Qin, L. P., Zheng, H.C. and Rui, Y.C. (2004). Journal of Chinese Integrative Medicine, 2 (6), 440-444. (2) Li, H., Gong, X., Zhang, L., Zhang, Z., Luo, F., and Zhou, Q. (2009). Phytomedicine, 16 (6-7), 538-546.

\section{METABOLIC EFFECTS OF CITRUS GRANDIS WHOLE FRUITS EXTRACT IN THE STREPTOZOTOCIN-INDUCED DIABETIC RATS}

\section{(C) Raasmaja Atso ${ }^{1}$, Kovaleva Maria A. ${ }^{2}$, Makarova Marina N. ${ }^{2}$, Li Xiang Ming ${ }^{3}$, Zou Jianqiang ${ }^{3}$, Zhu Guo-Guang ${ }^{3}$, Pozharitskaya Olga N. ${ }^{2}$, Makarov Valery G. ${ }^{2}$, Shikov Alexander N. ${ }^{2}$, Hiltunen Raimo ${ }^{4}$}

${ }^{1}$ Division of Pharmacology \& Toxicology, Faculty of Pharmacy, University of Helsinki, P. O. Box 56 (Viikinkaari 5E), FIN-00014 Finland. ${ }^{2}$ St-Petersburg Institute of Pharmacy, 47/5, Piskarevsky pr., 195067, St.-Petersburg, Russia.

${ }^{3}$ Guangdong Huazhou Green Life Co., Ltd., Huazhou City, Guangdong, PRC. ${ }^{4}$ Division of Pharmaceutical Biology, Faculty of Pharmacy, University of Helsinki, P. O. Box 56 (Viikinkaari 5E), FIN-00014 Finland

Citrus grandis fruits are used as a folk remedy in Korea for hangovers, peel of the fruit in Taiwan for the treatment of stomach ache, in Cameroon traditional medicine for treatment of hypertension, in Chinese medicine as antitussive, expectorant and antiasthmatic. Dried Citrus peels, known as Chenpi are used in 